bowel disease (IBD) patients. The project, entitle IBD-SSHAMP (Supported, Self Help And Management Programme), was funded by an Innovation Award presented by the East of England Primary Care Trust (PCT) Innovation Team. The aim was to discharge patients from routine clinic visits, whilst maintaining an efficient remote monitoring system that could be co-ordinated through our specialist nurses.

Methods The first stage was to ensure we had a complete and upto-date database containing all of our IBD patients. After a retrospective 10 year review we identified a total of 2790 IBD patients, from 19 different ethnic backgrounds. Of these, 26 patients lacked mental capacity with learning disability or dementia and 117 did not have internet access. 370 of our original cohort had died by the time of commencing the project. Using Patient Knows Best we developed individualise websites to offer a communication portal between patients and specialist care, through which we could monitor their symptoms and offer management advice through a traffic light system. An alert is sent out to the IBD nurses and clinician involved if any patients symptom indices deteriorate markedly. Periodic faecal calprotectin and inflammatory markers will also be used to support the monitoring process. Virtual clinics will be held for these patients twice a year. If necessary patients can be seen in hospital clinics usually within $24-48 \mathrm{hrs}$.

Results Of the available 2,277 IBD patients, we have successfully discharged 400 onto the first wave of IBD-SSHAMP, with a further 300 due to follow shortly in the second wave. With confidence in the system building amongst the relevant clinicians the second wave will primarily contain patients stable on immunosuppressants eg. azathioprine. With most patients being seen at 6 monthly intervals, and follow up clinic appointments costing our PCT £85, this project could potentially save them $£ 119,000$ per year, whilst still providing a patient friendly and efficient management system. Conclusion IBD-SSHAMP is the UK's first internet based remote management system for managing stable IBD patients. It aims to reduce cost and free up NHS outpatient time, whilst providing an efficient monitoring and management programme. This is a proof of concept project, from which further data outcomes will be presented.

Disclosure of Interest None Declared

\section{OC-081 DEFINING CIRRHOSIS WITH FIBROSCAN FOR ENTRY TO HEPATOCELLULAR CARCINOMA SURVEILLANCE IN CHRONIC HEPATITIS C: A UK COST EFFECTIVENESS ANALYSIS}

doi:10.1136/gutjnl-2013-304907.080

1." C Canavan, ${ }^{2} \mathrm{~K}$ Corey, ${ }^{2,3} \mathrm{C}$ Hur. ${ }^{1}$ Division of Epidemiology and Public Health, University of Nottingham, Nottingham, UK; ${ }^{2}$ Gastrointestinal Unit; ${ }^{3} / n s t i t u t e$ for Technology Assessment, Massachusetts General Hospital, Boston, United States

Introduction Chronic hepatitis $\mathrm{C}(\mathrm{HCV})$ is a significant risk factor for cirrhosis and subsequently hepatocellular carcinoma (HCC). HCV patients with cirrhosis are screened for HCC every 6 months. Surveillance for progression to cirrhosis, and consequently access to HCC screening, is not standardised. Liver biopsy, the usual test to determine cirrhosis, carries a risk of significant morbidity. Ultrasound elastography (Fibroscan) is a non-invasive test for cirrhosis. This study assesses the cost effectiveness of annual surveillance for cirrhosis in chronic HCV and the effect of replacing biopsy with fibroscan to diagnose cirrhosis.

Methods A Markov decision analytic model simulated a hypothetical cohort of 10000 patients with chronic HCV initially without fibrosis over their lifetime. Cirrhosis surveillance strategies assessed were: (A) no surveillance; (B) current practise; (C) fibroscan in current practise with biopsy to confirm cirrhosis; (D) fibroscan completely replacing biopsy in current practise (definitive); (E) annual biopsy; (F) annual fibroscan with biopsy to confirm cirrhosis; (G) annual definitive fibroscan.
Results The model was calibrated with good visual fit. Annual definitive fibroscan is the optimal strategy choice. Sensitivity analysis shows this outcome to be robust. The cost-effective frontier holds strategies $\mathrm{A}$ and $\mathrm{G}$ with $\mathrm{E}$ dominated by extension. All other strategies are strictly dominated. It diagnoses $20 \%$ more cirrhosis than the current strategy, with 549 extra patients per 10000 accessing screening over a lifetime; consequently 76 additional HCCs are diagnosed. Lifetime cost is an additional $£ 98.78$ per patient compared to current strategy for an additional 1.72 unadjusted life years. Annual fibroscan surveillance of 132 patients diagnoses one additional HCC over a lifetime. The ICER for annual definitive fibroscan is $£ 6557.06 /$ QALY gained.

Conclusion Annual definitive fibroscan may be a cost-effective surveillance strategy to identify cirrhosis in patients with chronic HCV to allow access to HCC screening.

Disclosure of Interest None Declared

\section{OC-082 THE DEVELOPMENT OF A WEB-BASED SYSTEM TO ENHANCE CARE IN INFLAMMATORY BOWEL DISEASE}

doi:10.1136/gutjnl-2013-304907.081

1. ${ }^{*} \mathrm{C}$ R Calvert, ${ }^{2} \mathrm{~S}$ Lal, ${ }^{2} \mathrm{C}$ Stansfield, ${ }^{1} \mathrm{~J}$ McLaughlin, ${ }^{2} \mathrm{~A}$ Robinson. ${ }^{1}$ School of Translational Sciences, Manchester University, Manchester; ${ }^{2}$ G.I. Department, Salford Royal Foundation NHS Trust, Salford, UK

Introduction Engaging and empowering patients to have greater involvement in their care is central to the vision of many governmental health policies. It is widely recognised that facilitating selfmanagement for patients by providing information to help support understanding, decision-making and motivating behavioural change can lead to significant improved health outcomes. ${ }^{1}$ We describe the development of a web-based IBD system that specifically aims to support patients' in taking a more active role in managing their IBD. Methods Design and implementation of a web-based system in the healthcare setting is complex with a number of human, technological and organisational factors that need to be considered from the start of the concept. Stakeholder involvement is essential throughout this process. Using the theoretical framework of self-efficacy we have designed a system with the specific aim to support patients to engage in their health. Our aim has been to develop a web-based system that is easily managed and sustainable for use in routine clinical practise.

Results 'My IBD Portal' has now been implemented. The webbased system is accessed via a secure login and provides patients with a number of components. This includes a summary of their disease, clinic letters and their latest test results. Functions known to further enhance self-management are included with electronic self-monitoring of symptoms and decision support. Secure messaging with the IBD unit is provided. Throughout the site personalised and trusted information links are integrated. The system is automated to populate the specific data fields each day from the hospital electronic medical record. The infrastructure of the system, has been specifically designed to be easily adopted throughout the UK healthcare setting.

Conclusion Design and implementation of patient-focused ehealth within the NHS is complex. Stakeholder involvement is essential. Evaluation of web-based interventions requires careful consideration, as they are often complex interventions by nature. The revised MRC framework provides a useful resource to develop a systematic process to evaluate such technologies. ${ }^{2}$ We are currently evaluating the impact of 'My IBD Portal' in a formal trial setting.

Disclosure of Interest None Declared

\section{REFERENCES}

1. De Silva et al. Evidence:Helping people help themselves. The Health Foundation 2011. http://www.health.org.uk

2. Anderson et al. New MRC guidance on complex interventions. 2008. BMJ; 338:a1937 